\title{
Etnografía y políticas públicas en materia de justicia y violencia contra las mujeres en Chile
}

\author{
Fabiola Miranda Pérez \\ Universidad Alberto Hurtado, Santiago, Chile. \\ Email: fmiranda@uahurtado.cl \\ Daniella Gac Jiménez \\ Universidad de los Lagos, Santiago, Chile. \\ Email: daniella.gac@ulagos.cl
}

\begin{abstract}
Resumen: El presente análisis busca reflexionar sobre los aportes de la etnografía al estudio de las políticas públicas utilizando como ejemplo, el caso de la intervención estatal en materia de violencia contra las mujeres en Chile en la última década. Para lo anterior, se expone aquí un trabajo etnográfico realizado en Tribunales de Familia en la Región Metropolitana, siguiendo casos de violencia conyugal, que combina observación directa con observación participante. Se sugiere la idea que la metodología etnográfica resulta substancial a la hora de aprehender la fase de implementación de las políticas públicas, ya que permite develar problemáticas y desigualdades que van más allá de aquellas que se presentan en el contacto entre el Estado y los/as usuarios/as, revelando además conflictos de legitimidad internos que las metodologías más clásicas de la Ciencia Política de corte top down no hubiesen a priori sacar a relucir. De esta manera se concluye que la etnografía no sólo se rinde cuenta de las modalidades de intervención, sino que también se avanza en el conocimiento del «Estado en acción» lo cual permitió mejorar la comprensión de las barreras que ha tenido desde la Justicia el estudio de las violencias contra las mujeres en este país.
\end{abstract}

Palabras clave: Violencias contra las mujeres; tribunales de familia; etnografía; políticas públicas; ciencia política.

\section{Ethnography and public policies regarding justice and violence against women in Chile}

\begin{abstract}
The present analysis aims at thinking about the contributions of ethnography to the study of public policies, using as an example, the case of state intervention concerning violence against women in Chile in the last decade. To do so, we present here an ethnographic study carried out in Family Courts in the Metropolitan Region, following cases of domestic violence, which combines direct observation with participant observation. We suggest the idea that the ethnographic methodology is substantial when it comes to apprehending the implementation phase of public policies, since it allows to reveal problems and inequalities that go beyond those presented in the contact between the State and the users, revealing also internal conflicts of legitimacy that the more classic methodologies of Political Science of top down nature would not have a priori brought to light. In this way, the conclusion is that ethnography not only gives an account of the modalities of intervention, but also advances in the knowledge of the «State in action», which has allowed a better understanding of the barriers that the Justice Department has had in the study of violence against women in this country.

Keywords: Violence against women; family courts; ethnography; public policies; political science.
\end{abstract}

\section{Etnografia e políticas públicas de justiça e violência contra as mulheres no Chile.}

Resumo: A presente análise busca refletir sobre as contribuições da etnografia para o estudo de políticas públicas, utilizando como exemplo, o caso da intervenção estatal na violência contra a mulher no Chile na última década. Para o anterior, se apresenta aqui um trabalho etnográfico realizado nos Tribunais de Família da Região Metropolitana, após casos de violência conjugal, que combina observação direta com observação participante. Sugere-se que a metodologia etnográfica 
seja substancial quando se trata de apreender a fase de implementação das políticas públicas, pois permite salientar problemas e desigualdades que vão além daqueles que surgem no contato entre o Estado e os/as usuários/as, revelando também conflitos de legitimidade interna que as metodologias mais clássicas da ciência política do tipo top down não teriam a priori trazido à luz. Dessa forma, conclui-se que a etnografia não apenas realiza as modalidades de intervenção, mas também avança no conhecimento do «Estado em ação», o que permitiu melhorar a compreensão das barreiras que desde a Justiça vem enfrentando o estudo das violências contra as mulheres neste país.

Palavras-chave: Violência contra a mulher; tribunais de família; etnografia; políticas públicas; Ciência Política.

\section{Introducción}

El presente trabajo se origina de la reflexión acerca de la metodología utilizada y los resultados obtenidos en un trabajo anterior y de la necesidad de ampliar y profundizar dichos resultados a partir de la integración de una nueva metodología de análisis, la etnografía. Dicho trabajo se enmarcó, de manera general, en un análisis de política pública desde el enfoque de la Sociología de la acción pública (Commaille, 2010) para reconocer el rol jugado por los actores, en este caso agentes estatales, en la implementación de las políticas públicas ${ }^{1}$. El objetivo de este análisis fue conocer cómo se configuran y reconfiguran los mecanismos de tratamiento de las violencias contra las mujeres en el Chile actual, desde un estudio a los organismos de protección social como lo fue el Servicio Nacional de la Mujer ${ }^{2}$ y al interior de instituciones de justicia, específicamente, los Tribunales de Familia, Tribunales de Garantía y el Ministerio Público.

Se plantea, a grandes rasgos, que las políticas públicas de tratamiento de las violencias hacia las mujeres responden a las lógicas de un Estado subsidiario, focalizando los recursos públicos, presupuestales, cognitivos, infraestructura, entre otros; como señalarían Subirats et al (2008), en una población caracterizada por su vulnerabilidad social. Desde esta construcción, se comprobó que los agentes de la intervención y/o implementación contribuyen a replicar ciertos constructos sociales sobre las y los destinatarios de la acción pública, basados muchas veces en prejuicios que se construyen de la asignación de distintas relaciones de poder (Crenshaw, 1989) que se manifiestan en las interrelaciones entre los agentes intervinientes y los destinatarios de la política pública.

El trabajo, desde una perspectiva epistemológica, buscó conocer de manera integral la política pública de lucha contra las violencias de pareja en Chile entre los años 2005 y 2015, eligiendo para aquello una metodología cualitativa de análisis cognitivo de la acción pública (Surel, 2010), específicamente en la etapa de implementación de políticas públicas. Así, se indagó sobre las percepciones de los actores encargados de la intervención directa con las mujeres víctimas del maltrato, es decir, se buscó conocer cómo la esfera pública da tratamiento a lo privado. Resulta importante destacar la complejidad del sujeto de estudio, desde ahí, que tempranamente se definió como eje principal de análisis al Estado debido a que el trabajo se inscribe disciplinarmente en la Ciencia Política y en la Sociología Política.

El posicionamiento epistemológico y metodológico se relaciona con tres dimensiones que se consideraron claves en el abordaje de la problemática. La dimensión socioeconómica de los y las destinatarias de la acción pública, la dimensión subjetiva de las y los agentes de la intervención, y la dimensión sociopolítica relativa al contexto sobre el cual se estudia. De esta manera, se utilizó una metodología integradora: el enfoque social comprensivo de Weber (2006), que se posiciona también en la interpretación con el fin de acercarse a los espacios sociales donde se llevan a cabo los dispositivos de acción y control social.

Con la finalidad de entender la complejidad de los espacios sociales se siguió la línea de la fenomenología de acción social de Alfred Schütz (Blin, 1999), para lo que resulta crucial destacar la interacción social. Así, desde un enfoque de construcción social, se buscó aprehender prácticas cotidianas de los actores en sus espacios sociales - en este caso en instituciones de justicia- con la finalidad de entregar una descripción analítica del sujeto de estudio, tanto en el marco de su emergencia como al interior de su estructura. 
Durante la investigación se presentó como desafío, encontrar una metodología que fuera pertinente para crear un corpus sólido sobre los estudios sobre la implementación de las políticas públicas, los cuales, si bien están en aumento, aún no entran en el mainstream, como los de diseño de programas públicos o de evaluación final (Revuelta, 2007). Otra problemática, referida a la metodología empleada, fue la selección del instrumento de recolección de datos; en este caso: la entrevista semi-estructurada de carácter cualitativa (ampliamente utilizada en Ciencia Política). En el análisis de los resultados se determinó que la entrevista como instrumento, presentó límites claros al no evidenciar cómo muchos y muchas de los y las agentes estatales entrevistados/as (sesenta y siete) se posicionaron sobre su quehacer. Para lo cual es necesario integrar metodologías complementarias que permitan aproximarse de manera comprensiva al sujeto de estudio.

A partir de lo anterior, el presente trabajo sugiere la idea que la metodología etnográfica resulta substancial a la hora de aprehender la fase de implementación de las políticas públicas (Revuelta, 2007), ya que a través de ella es posible develar problemáticas y desigualdades que van más allá de aquellas que se presentan en el contacto entre el Estado y los/as usuarios/as. Otro elemento fundamental es que permitirá determinar conflictos de legitimidad internos que las metodologías más clásicas de la Ciencia Política de corte top down (Sabatier y Mazmanian, 1980) no hubiesen a priori sacar a relucir. De esta manera, el enfoque de análisis cognitivo de política pública, es decir, aquel que se preocupa de las percepciones que tienen los actores intervinientes de la política pública, que se relaciona con aspectos culturales sobre la concepción ellos mismos y del mundo (Muller, 2000), plantea la necesidad de utilizar metodologías que vayan más allá del formalismo de la entrevista sociológica de corte más clásico. Así, un desafío importante es la comprensión del sujeto de estudio como un conjunto en donde se engarzan aspectos formales a partir de un discurso ético-político considerado adecuado por quienes lo emiten; pero también prácticas y reflexiones en el quehacer cotidiano de los actores que van develando aristas ético-sociales que determinan la intervención que ejerce en este caso el Estado hacia las personas que orienta su acción. En consecuencia, el enfoque cognitivo de política pública se alimenta de metodologías sociológicas que permitan conocer en profundidad el origen de las interacciones y del saber-hacer estatal.

Con el propósito de ampliar lo evidenciado en la investigación anterior y utilizando e incorporando la metodología de investigación cualitativa de la etnografía, es que el presente estudio pretende profundizar y complementar la reflexión preliminar acerca del «Estado en acción» (Palier y Surel, 2005). Todo esto a través del análisis de las observaciones realizadas en Tribunales de Familia y penales en Chile en la Región Metropolitana de Santiago, de audiencias relativas a casos de violencia de pareja durante el año 2013 entre los meses de octubre y diciembre, y entre los meses de marzo a mayo 2014 respectivamente ${ }^{3}$.

En este escrito se presentarán diversos puntos de discusión referidos a la aplicabilidad de la etnografía como metodología de análisis en las ciencias sociales y las políticas públicas, presentando los antecedentes sobre el tratamiento de parte del Estado sobre la violencia hacia las mujeres. Así como se integrarán al análisis preliminar, nuevos elementos que permitirán el establecimiento de relaciones que en la investigación anterior no han sido profundizados.

\section{Justicia y violencias contra las mujeres en Chile: Antecedentes del estudio}

En Chile el Estado intervino en materia de violencias contra las mujeres desde inicios de los años '90 a partir de la creación de la primera Ley de Violencia Intrafamiliar o VIF (N 19.325) el año 1994. Esta ley da la categoría de falta al delito de violencia intrafamiliar, siendo esta materia conocida por los tribunales civiles. Luego de importantes críticas de la sociedad civil y de estudios que mostraban el bajo impacto de la ley, sobre todo en las mujeres denunciantes (Miranda \& González, 2018); un grupo conformado por diputadas de centro izquierda (a inicios del año 2000) propusieron un nuevo texto al parlamento con el fin de mejorar la ley anterior y de presentar esta temática como una prioridad para el Estado, destinando recursos para que las violencias no sigan siendo invisibilizadas.

De manera paralela, en el país se vivían procesos importantes de reformas a la justicia en el ámbito familiar y en el sector penal (Azócar y Ferree, 2015; Hersant, 2017). En materia de familia surgieron en el año 2004 los Tribunales de Familia, creándose una justicia especializada en esta esfera y una manera especial de intervenir 
desde la justicia las problemáticas relativas a los conflictos familiares. En tanto, en el ámbito penal, la reforma también es profunda, ya que se modificó todo el proceso de un modelo inquisitivo a un acusatorio, poniéndose en marcha nuevos tribunales: Tribunales de Garantía y el Tribunal oral en lo Penal. De manera accesoria y para completar dicho proceso, se implementó en 2005 el Ministerio Público, que se especializa en la representación a víctimas de delitos y en algunos casos en su intervención. La nueva ley VIF, aprobada en 2005 y vigente hasta nuestros días, captura ese cambio institucional, involucrando no solo al Servicio Nacional de la Mujer -quién pilota la nueva política pública- sino que a todos estos sectores de justicia (Miranda Pérez, 2017).

La justicia familiar, se encarga de conocer, de orientar y de fallar en los casos de violencia psicológica, desde una perspectiva integral ya que en estos tribunales se incorpora un cuerpo psicosocial de asesoría a los jueces el Consejo Técnico, compuesto por psicólogos y trabajadores sociales (Miranda Pérez \& Riveros, s.f.). En la justicia penal, la creación del delito de maltrato habitual y la consideración de otros delitos en contexto intrafamiliar como lesiones y amenazas, obliga a este órgano a recibir estos casos (Villegas, 2012). En tanto el Ministerio Público, recibe denuncias y también de acompañar junto a sus profesionales psicosociales los casos más complejos a través de la Unidad Regional de Atención a Víctimas y Testigos (URAVIT) (Miranda Pérez, 2017).

Es bajo todas estas nuevas configuraciones que en esta investigación se pretende en primer lugar, determinar cómo se implementa a nivel de la justicia la política pública el tratamiento integral de las violencias contra las mujeres; conocer quiénes juegan un rol clave en la puesta en marcha; y finalmente, conocer la valoración que dicha temática adquiere para las y los implementadores de la política pública.

\section{Etnografía en Ciencia Política y Políticas públicas}

Las investigaciones cualitativas se basan en métodos de formulación de datos que se deben adaptar a las condiciones y al contexto social en las que ellas operan (Glaser y Strauss, 1967). De este modo, las investigaciones de campo o de terreno se han ido transformando en una necesidad, para la comprensión real de los fenómenos, es decir, para aprehender la complejidad y variabilidad del comportamiento humano. Para ello, el conocimiento de las relaciones entre las condiciones (estructuras) y las acciones (procesos) se devela esencial. Dado lo anterior, la misión del investigador es observar con atención lo que pasa, tomar notas detalladas y cuestionar a los y las encuestados/as para poder comprender el objeto, la razón y la intención y/u objetivo de su acción. Dicho de otro modo, el investigador a través de las interacciones con su campo construye de manera activa conocimiento.

Históricamente, el trabajo etnográfico ha estado relacionado a las investigaciones de la Antropología y en segundo lugar aquellas de la Sociología. Sin embargo, desde hace ya algunas décadas, de manera creciente un número importante de investigaciones sociológicas han utilizado la etnografía (Hammersley y Atkinson, 2007), sobre todo aquellos estudios sobre los procesos de urbanización y de industrialización (Ibíd.). A mediados de la década del '50, los sociólogos de la Escuela de Chicago se focalizan en los case studies, para quienes la observación etnográfica se reveló como un enfoque necesario en la comprensión de la vida social de los individuos estudiados. Así, este método comenzó a ser utilizado por disciplinas como la Psicología y la Geografía, y más tarde se abriría a la Ciencia Política, principalmente a través de estudios teóricos que se inscriben en corrientes constructivistas. Esta tendencia se desarrolla en Estados Unidos, donde sociólogos y antropólogos intentaron crear una subdisciplina denominada political ethnography (Joseph et al., 2007). La idea de aquello es rechazar la mirada estatocéntrica weberiana que busca racionalizar la acción de quienes intervienen en ella, entendiendo a la política como un «hecho social» (Sharma y Grupta, 2006:8) mediada por condiciones contextuales y culturales que influyen en los comportamientos de los agentes estatales.

Pese a que la Ciencia Política parecía no concernida, algunos trabajos recientes defienden el uso de la etnografía, notoriamente aquellos que tratan el estudio de políticas públicas (Shatz, 2013)4. Así, se invita a pensar al Estado no como una construcción o ilusión (Franzé Mudanó, 2013) sino como un conjunto de prácticas con efectos concretos que condicionan los resultados esperados de su intervención. De esta manera, la etnografía del Estado se ha transformado en una corriente metodológica que se amplía cada vez más a diferentes disciplinas (Dubois, 2018; Rojas Lash, 2019). 
En lo que concierne al estudio de las violencias contra las mujeres y la respuesta estatal desde los órganos de justicia, un método de observación mixto se impone durante la investigación. En efecto, en algunos momentos se realizan observaciones directas sin interacción con el sujeto destinatario de la política pública; y en otras ocasiones, debido a la solicitud de los agentes estatales observados, se realizan observaciones participantes debido a la invitación de los agentes observados de cooperar con sus acciones durante dicho proceso. De esta manera, la observación «no se limita solo a la mirada. Ella implica también un doble esfuerzo de sistematización y de transcripción de los fenómenos observados» (Coman et al., 2016, p.181-182). El trabajo se traduce entonces en la toma de notas, pero también en conversaciones informales y en la posibilidad de interactuar con un abanico amplio de actores durante el proceso de implementación.

En este sentido, la importancia y pertinencia de la etnografía para el estudio de las políticas públicas, sobre todo de aquel que se autoproclama de cognitivista, radica en que la observación resulta útil «para analizar los comportamientos individuales y las interacciones entre los individuos al interior de los grupos» lo cual permite aproximarse a «la formulación y las condiciones de puesta en marcha de una política pública» (Coman et al., 2016. P. 182). No obstante, es importante destacar que:

«Como en toda investigación, la observación está al servicio de una construcción de un objeto: ella selecciona solo una parte de la realidad de la cual los limites están trazados por una problemática. Toda vez que ella lleva a aprehender de esta parte como una totalidad concreta: ella invita al investigador a considerarse como un elemento de esta totalidad, y a analizar los efectos de su presencia más que intentar neutralizarla a cualquier precio» (Chauvin y Jounin, 2012, p.144).

De esta manera, el método etnográfico presenta una serie de ventajas para entender la implementación de una política pública, y es importante indicar que la presencia de un investigador no es neutra para los actores observados, ni para la interacción que se desarrolla durante la observación. Si lo anterior pudiese tener en algunos casos efectos notables, la ventaja que tiene la observación sobre la realización de entrevistas, es que en general, los agentes observados, tienen la percepción de no estar transmitiendo alguna opinión o mensaje que comprometa su trabajo. Debido a que la interacción se sitúa en un contexto donde los actores no se sienten obligados y/o expuestos a justificar su proceder todo el tiempo; dando paso al trabajo de interpretación por los investigadores de lo observado en un momento y lugar determinado.

En ese sentido, es que este estudio intentó reflexionar tanto en los factores endógenos como exógenos de la asistencia social en Chile; y en particular, del tratamiento judicial de las violencias contra las mujeres. Para ello, esta investigación se funda en una base empírica primaria y secundaria, que pudimos obtener gracias a la utilización de distintos instrumentos de investigación cualitativa, en donde la etnografía se inserta como una metodología que nos permite captar la realidad social desde un enfoque comprensivo ${ }^{5}$.

\section{Estudiar el cotidiano al interior de los tribunales: otros aspectos metodológicos}

Como lo señala Baudouin Dupret, de manera general las investigaciones sociológicas sobre el derecho y la justicia han intentado explicar fenómenos jurídicos en términos de relaciones de poder y de dominación, como una «traducción simbólica de una cultura interiorizada» (Dupret, 2010, p. 316), considerando al «derecho tal como es, una actividad práctica» (Ibíd.). Sin embargo, consideramos con esta nueva proyección ampliar y profundizar el análisis de los usos del derecho, por parte de los agentes del Estado que trabajan al interior de los tribunales de familia, a través del estudio de sus «rutinas» (Mazouz, 2014). En consecuencia, el objetivo de la nueva propuesta con enfoque etnográfico fue situar las prácticas de los operadores de la justicia en el seno de los tribunales de familia para «ver cómo las actividades se organizan y cómo las personas se orientan en relación a las estructuras de sus actividades» (Mazouz, 2011, p. 330).

La aplicación de normas jurídicas lleva a considerar el derecho según cómo sus normas fueron elaboradas, pero también a dilucidar el carácter dinámico de los valores que expresan las normas y que manifiestan las tensiones, contradicciones y dificultades que ellas encuentran en la práctica. Los problemas que surgen en la aplicación de las leyes se asocian rápidamente a la ineficacia de ellas y a la falta de 
visión del legislador, o bien a la incapacidad de las instancias implementadoras de cumplir con los objetivos propuestos en la ley.

Ahora bien, ¿qué pasa cuando una ley no satisface las expectativas de las personas?, ¿de qué manera los actores de la implementación, cuando surgen estos obstáculos, intentan poner en marcha prácticas y procedimientos que ayuden a las víctimas de las violencias de pareja en este caso?, ¿cómo sobrellevar los procedimientos institucionales para entregar respuestas en un corto plazo?

Desde nuestro punto de vista, ninguna de estas preguntas puede tener una respuesta sin estudiar el contexto y en específico las transformaciones de las instituciones de justicia y/o sin pasar por la utilización del enfoque etnográfico. Este último al ser dinámico y al revelar interacciones, muestra al Estado como un conjunto de relaciones sociales insertas en estructuras de poder. Dichas estructuras van permeando la forma de tratar los problemas públicos. Por aquello, se exploran las prácticas, las percepciones y las visiones (Mazouz, 2011), considerando todo lo que podría conceptualizarse como violencia de pareja, desde el discurso y las acciones de los agentes de la intervención directa con los destinatarios: trabajadores sociales, psicólogos, abogados, jueces y otros operadores de justicia 6 .

La influencia que ejercen dichos actores en la aplicación de las normas es determinante para establecer la intervención del Estado y la forma por la cual son tratados los casos que llegan a esta instancia de justicia. Se trata entonces de considerar a los policy-makers, a través del «ejercicio de su poder de apreciación, en lo que concierne a las prestaciones sociales de un estatuto o de una acción de control, ellos le entregan el sentido a la acción pública o bien hasta invertir su sentido» (Delpeuch et al., 2014, p. 167).

Para ello, se presenta cómo el Poder judicial y otros organismos de justicia a través de sus actores dan paso a prácticas y a interpretaciones particulares sobre el fenómeno de las violencias de pareja. En este sentido, el concepto de campo es uno de los ejes que estructura este trabajo. Los trabajos que tratan específicamente sobre el «campo judicial» según las nociones y reflexiones aportadas por Pierre Bourdieu (1986) constituyen también un antecedente teórico importante.

Este autor propone un análisis que reposa sobre la noción del habitus judicial, las luchas por el capital simbólico y el reconocimiento, que resulta pertinente al sujeto de las violencias. La justicia como institución sería entonces el espacio de competencia entre distintos agentes investidos de capacidades sociales y técnicas, que los habilitan a interpretar - no sin conflictos- un corpus de textos legales, o bien a intervenir de manera directa a las personas concernidas por una problemática particular. Dado lo anterior, las partes se someten a aceptar nuevas interpretaciones, basadas en normas jurídicas y en prácticas cotidianas de la justicia, para resolver sus problemas en este caso de la esfera privada, poniendo en confrontación sus visiones con aquellas de un agente externo.

Para reconstituir las interacciones que surgían del tratamiento de los tribunales de las violencias de pareja, se siguieron de manera aleatoria algunos casos de mujeres afectadas por las violencias hasta la obtención de una primera respuesta por parte del tribunal ${ }^{7}$.

\section{La Observación Etnográfica y los desafíos al estudio de los tribunales en acción}

Dado que el trabajo se sitúa en una reflexión acerca de la pertinencia del enfoque etnográfico para el estudio de las políticas públicas que implementa la justicia en el tratamiento de los casos de violencia de pareja, se expondrán las reflexiones e interpretaciones de los investigadores asociadas a las dificultades del estudio y/ o hallazgos surgidos.

Tal como se señala más arriba, se utilizó un método mixto de recolección de datos, proceso que se inició con entrevistas semiestructuradas con un conjunto importante de funcionarios y funcionarias estatales, ergo, nos concentraremos en aquellos del ámbito judicial donde se hicieron las observaciones. 
Investigar sobre los casos de violencia de pareja resultó un proceso complejo que implicó ganar confianza previa con los agentes que trabajan cotidianamente en ello, lo que se logró gracias a una serie de entrevistas previas y de reuniones en donde se fueron negociando los espacios otorgados para la investigación. Los argumentos esgrimidos para tal desconfianza surgían de la naturaleza misma de la intervención de la jurisdicción familiar que trata asuntos relativos al orden privado de las personas.

Otra de las principales resistencias tuvo que ver con la disciplina en la cual se enmarcaba el estudio: no se trataba de un estudio legal, ni sobre mejoras al modelo de intervención psicosocial, sino que se trataba de entender el funcionamiento de una política pública desde la Ciencia Política, en una lógica en donde los actores no se sentían policy-makers, sino más bien aplicadores de ley.

Lo anterior, tuvo como consecuencia un largo periodo de negociación entre las entrevistas y la realización de las observaciones en sí. Sin embargo, una vez obtenido el acceso a un tribunal, el siguiente tribunal observado consideró este antecedente como una prueba de confianza que nos permitió integrarnos rápidamente en él.

En efecto, las entrevistas fueron un instrumento que nos permitió abrir las puertas para entrar a los tribunales de familia en una primera instancia, y luego, para poder asistir junto con un miembro del Ministerio Público a audiencias en el ámbito penal ${ }^{8}$. Dicho de otro modo, las entrevistas dieron la oportunidad de ingresar algunos meses más tarde en el quehacer cotidiano de un dominio hermético de las y los abogados.

Inspirándonos en trabajos sobre espacios administrativos realizados en Francia (Spire, 2005; Le Caisne, 2007), pudimos, gracias a las observaciones etnográficas, descifrar espacios que las entrevistas no eran capaces de mostrarnos. El conocimiento de las interacciones cotidianas, las representaciones movilizadas por los actores, conocer los márgenes de discrecionalidad (Muller et al., 1996), más allá de aplicación de la legislación. En efecto, la observación etnográfica nos permitió tener una perspectiva comprehensiva del trabajo realizado por las instituciones de justicia, porque ella permite «establecer el vínculo entre los hechos [...] observador y las particularidades del contexto en que estos se llevan a cabo» (Dodier y Baszanger, 1997, p. 37).

En cuanto al ámbito familiar, que es al cual haremos alusión de manera principal en este artículo, tuvimos la oportunidad de realizar observaciones en dos grandes Tribunales de la Región Metropolitana de Santiago entre los meses de octubre a diciembre 2013. El primero de aquellos abarca una cantidad importante de comunas del sector sur de la capital y el segundo, de mayor envergadura, comunas tanto del sector norte como del sector oriente de la capital.

Tanto las entrevistas como las observaciones nos permitieron complementar y profundizar los análisis previos, ya que pudimos determinar el territorio y las prácticas, además del relevamiento del pensamiento crítico de los actores.

\section{Prácticas y territorio}

Desde la observación etnográfica se evidencia que cada Tribunal de Familia, cuenta con una organización distinta en cuanto a los protocolos internos de funcionamiento, como en relación a la forma en que se sistematizan las denuncias y se organiza a su personal. Prácticas variables que dependen, desde nuestro punto de vista de la cultura organizacional forjada al interior de cada uno, pero también en consideración a la cantidad de población que cada uno de ellos cubre.

Dicho de otro modo, y utilizando un ejemplo, cada tribunal dependiendo de su equipo y de su contexto decide en conjunto la metodología de recepción de las denuncias que será privilegiada. Así, se percibe que el método de recepción de denuncias influye en la judicialización de los casos. En efecto, en el tribunal que cubre un espectro menor de población, se privilegia el «método presencial» donde la persona se acerca al tribunal para

contar su historia, favoreciendo la posibilidad de obtener una respuesta por parte de la justicia de manera más rápida. Incluso si su nivel de riesgo no era muy elevado según los resultados de la pauta de evaluación ${ }^{9}$ de éste, 
la cual era establecida por los miembros del Consejo Técnico. Así, muchas personas partían en la misma mañana con una medida de protección, por ejemplo. Tendemos a pensar que el contacto directo entre los actores de justicia y el público influía en ello.

Por otra parte, en el tribunal con una demanda mayor donde se privilegiaba la metodología de denuncia no presencial, vía telefónica, el procedimiento era más lento y centrándose más en la pauta de riesgo diseñada por este tribunal más que en el caso precedente. Por último, indicar que luego de este primer contacto con la víctima, si su denuncia era «seleccionada» por algún miembro por parte del Consejo Técnico, de igual modo sería citada para una entrevista personal con otro miembro del Consejero Técnico. Este último, tiene por tanto la responsabilidad de velar por que las causas que fueran a audiencia fueran las más plausibles.

Entonces, gracias a las técnicas propias de la etnografía, se observa que existen espacios de discrecionalidad para el acceso a la justicia que varían según los métodos e instrumentos aplicados por cada tribunal y que están directamente relacionados con la cantidad de población cubierta y con las características del territorio.

\section{Una problemática sensible pero de baja valoración}

Una vez establecidas las confianzas, comienzan a surgir valoraciones que iban más allá del aporte de nuestro trabajo, a reflexiones sobre la poca influencia que tenían las acciones de los funcionarios sobre la vida de las mujeres: el sujeto a intervenir, en este caso mujeres víctimas de violencia, muchas veces se presenta como el principal obstáculo para la consecución de los fines de la ley y de la política.

Muchos de los operadores de justicia, con los cuales se pudo interaccionar: jueces, trabajadores sociales, psicólogos, abogados etc, si bien pueden tener un nivel de compromiso importante en materia de tratamiento de las violencias intrafamiliares destacan que el desistimiento de las denuncias es un problema común y recurrente que afecta el normal desarrollo de las investigaciones e incluso dificulta cualquier tipo de intervención por parte de los profesionales «auxiliares de la justicia» ${ }^{10}$.

Otro elemento que llama la atención durante la investigación es la actitud de los funcionarios frente a las mujeres víctimas de la violencia de pareja. En efecto, existe una visión compartida de que la mayoría de los casos de violencia intrafamiliar son judicializados, aunque no deberían serlo, por tanto, se tiende a minimizar la importancia de éstos.

«En general yo creo que [la violencia intrafamiliar] es como [la temática]...quizás...que tiene menos relevancia jurídica [para los tribunales de justicia] podríamos decir [...] la percepción en general es que es como «Sala de Parejas», no sé si has visto ese programa de televisión de la doctora Polo (risas), es más o menos eso, la violencia intrafamiliar es como un poco eso... Lo otro, desde el punto de vista de persecución penal el hecho de que sea criminalizada está bien, pero hay una serie de delitos, una serie de comportamientos que sí constituyen delito y que debemos recibir aquí. Tenemos [los funcionarios] la sensación de que a [los tribunales de] garantía llega todo lo que no se pudo solucionar en otras instancias [haciendo alusión a los tribunales de familia] que sí a lo mejor debieron ser efectivas, a lo mejor sin judicializarlas». (Extracto de una entrevista a una jueza de garantía en Santiago, realizada el 5 de noviembre de 2013.)

Uno de los desafíos planteados por los propios funcionarios operadores de justicia es el reconocimiento de los discursos de las víctimas y su toma en consideración. Según lo declarado por algunos de los funcionarios, las violencias intrafamiliares implican problemáticas complejas, razón por la cual un número importante de agentes intentan implicarse lo menos posible con estos casos. Las características y perfil de este tipo de víctimas, como se destaca más arriba, contribuyen a la baja valoración que tiene la temática tanto en la jurisdicción familiar y aún más en el sector penal. 


\section{Profesionales legales y profesionales psicosociales}

Por último, resulta importante recordar que los tribunales de familia desde su creación cuentan con un cuerpo interdisciplinar especializado en familia: el Consejo Técnico, que se compone ya sea por Trabajadores Sociales y/o Psicólogos. Este cuerpo profesional «psicosocial», como se denomina a aquellos que participan en un campo de acción de lo social que se construye desde los años 1990, se destaca por una praxis socialcomunitaria que busca dar respuesta a un ideal de acción integral (Rojas, 2014).

El Consejo Técnico, desde su dominio de especialización participa en tanto que asesor de la decisión judicial. En el artículo 5 de la ley 19.968, que crea los tribunales de familia, se detalla que dentro de sus funciones está la de aconsejar, individual o colectivamente a los jueces en el análisis y mejor comprensión de las causas que conocen en el dominio de su competencia.

En una entrevista de contacto para la realización de observaciones en un Tribunal de Familia, la persona responsable de atención público destacó el rol que ha ido adquiriendo el Consejo Técnico durante la última década, señalando, tal como se observa en el extracto, que dentro de las bases de la reforma familiar uno de los principales focos fue avanzar en materia de inmediación y transparencia. Este cuerpo asesor del juez comienza a aumentar su protagonismo, contrariamente a lo que ella indica sucedía en los tribunales de menores donde los asistentes sociales se remitían a hacer informes sociales.

«[Los tribunales de familia] es una reforma basada en la oralidad, la inmediación, y la transparencia poniendo en el centro los derechos del niño desde las obligaciones que impone la Convención de los derechos del niño. [Esta reforma] también [incluye] la introducción de un profesional [psicosocial] en una especie de comité asesor del juez en la toma de decisiones en este sistema nuevo. Yo entré en el 2005 como consejera técnica, que sería como parte de este staff de [nuevos] profesionales, generalmente de psicólogos, asistentes sociales que se creó a partir de la reforma en los tribunales de familia. Antes existían los asistentes sociales en los tribunales de menores, pero su rol se limitaba a hacer informes sociales». (Entrevista de contacto con una responsable de atención de público de un Tribunal de Familia de la Región Metropolitana de Santiago, realizada el 23 de noviembre de 2012.)

En efecto, los consejeros técnicos son los encargados actualmente de la elaboración de informes que serán sometidos a conocimiento de los jueces de familia, quienes a través de ellos conocen las causas, incluso antes de que las partes o la víctima ingresen a la sala de audiencias. De esta manera, el rol del Consejero Técnico es de gran relevancia debido a que los criterios establecidos en el informe serán un soporte importante para el desenvolvimiento de los casos durante las audiencias y en las sentencias.

Lo anterior, marca una ruptura importante en el tratamiento de los casos de violencia de pareja en Chile, puesto que previamente a la existencia de estos profesionales y estos expertos psicosociales, las violencias eran tratadas por la justicia civil en donde interactuaban casi de manera total actores formados en derecho o bien formados dentro de la administración de justicia donde el juez tenía un rol central. No obstante, el rol de los jueces al interior de los Tribunales de Familia necesita ser re-analizado, porque pese a ser un actor decisivo y central, no es el único (Delpeuch et al., 2014).

Por otra parte, la especialización cada vez más creciente de profesionales expertos en materia de familia, y sobre todo de aquellas profesiones que completan el saber-hacer jurídico, como aquellas que poseen los miembros del Consejo Técnico, ha comenzado a jugar un rol importante y que requiere ser mejor analizado. Ahora, bien es necesario remarcar que a partir de la observación de las interacciones se puede determinar que la actividad jurisdiccional no se trata solamente de técnica jurídica (Delpeuch et al., 2014), por lo que resulta innegable plantear los espacios de discrecionalidad existentes dentro de la administración de justicia y de un análisis in fine de los agentes no formados en derecho.

Finalmente, expresar que gracias a la observación de estos actores se fueron planteando análisis de desigualdades que no solamente decían relación con la calidad y caracterización de los destinatarios de la acción pública, sino que además, se pudo comprender que los estigmas también se generan en el tipo de temáticas que 
trata las justicia, donde algunas cuentan con mayor valoración y otras con una baja legitimación tal como son los casos de violencia de pareja que pudimos acompañar durante las observaciones.

\section{Conclusiones}

A partir del punto anterior, se determina la pertinencia que adquiere en este caso el estudio a los tribunales desde un enfoque etnográfico de comprensión de la política pública. Muchos de los puntos esgrimidos no habían surgido desde el corpus de las entrevistas hasta que se llevaron a cabo las observaciones. Poder observar e interrelacionarse al interior de estos espacios abrió un mundo de cuestionamientos sobre el acceso a la justicia de las personas, que no solo estarían relacionados desde los atributos que las componen, sino por las causas que movilizan.

En un segundo lugar, resulta interesante para nosotros la utilización de la metodología etnográfica para entender de manera comprensiva las transformaciones de las instituciones de justicia en estos últimos años entendiendo que es un proceso que se co-construye de manera cotidiana desde una multiplicidad de actores tanto jurídicos como no jurídicos.

En tercer y último término, la comprensión del entramado institucional, a través de las observaciones, develó prácticas y situaciones que se presentan en la implementación de las políticas públicas que resultaron significativas de la acción social del Estado en materia de violencia contra las mujeres. Pudiendo, de esta manera, mostrar no solo la baja valoración de la temática por parte de los Tribunales de Justicia, sino que también los conflictos internos existentes entre la justicia familiar y penal, y entre los distintos Tribunales de Familia. Lo anterior nos lleva a plantear la presencia de distintas interpretaciones de la intervención estatal frente a la problemática, requiriendo por tanto un replanteamiento conjunto de las visiones generales, para poder desde allí, avanzar en estrategias territoriales más pertinentes y en adecuación a las necesidades de los destinatarios de la acción pública.

\section{Agradecimientos}

La investigación desde donde se origina el presente análisis corresponde a la tesis doctoral en Ciencia Política que tiene como título "Acción Pública y Justicia en el Chile postdictatorial. El tratamiento estatal de las violencias contra las mujeres» presentada en 2017 en la Universidad de Grenoble Alpes, Francia.

\section{Notas}

\footnotetext{
${ }^{1}$ El análisis se centra en una perspectiva cognitiva de política pública (Muller, 2000), que busca traducir los modos en que los actores usan, significan y se apropian de los referenciales de una sociedad en su quehacer cotidiano (Jobert y Muller, 1987).

${ }^{2}$ Actual Servicio Nacional de la Mujer y de la Equidad de Género (SERNAMEG).

${ }^{3}$ Cabe señalar que para el presente estudio nos vamos a referir de manera principal a las observaciones realizadas al interior de dos tribunales de familia situados al interior de la Región Metropolitana, en donde se hacen observaciones cotidianas. En el sector penal, se procede a acudir a audiencias públicas y a seguir de manera puntual el trabajo de un fiscal de flagrancia. Sin embargo, la sistematización del trabajo realizado en la sede de la justicia penal nos ayuda a comprender de manera más fina la implementación de la política pública de tratamiento y respuesta de las violencias contra las mujeres a este nivel.

${ }^{4}$ En efecto la Revista francesa de Ciencia Política publicó en 2007 un número temático titulado «Investigar en un medio difícil», vol. 57, $\mathrm{n}^{\circ} 1$, que se consagra de manera completa al tema de las investigaciones etnográficas en Ciencia Política. La misma revista por primera vez publica una crónica bibliográfica sobre las etnografías en su volumen 66 nº del año 2016.

${ }^{5}$ Entre estos instrumentos o técnicas: la explotación de fuentes secundarias, posibilitó la contextualización del tema de estudio y permitió definir dónde explorar de manera empírica. En segundo lugar, la realización de entrevistas semidirectivas (67 en total) con agentes del Estado que participaban en la implementación de la política pública ya sean en las esferas ejecutivas y judiciales (2012 y 2013); y en tercer lugar, la observación etnográfica, que se llevó a cabo en el seno de instituciones de justicia, a saber, dos tribunales de Familia de la Región Metropolitana y en tribunales penales de la misma región en los años 2013 y 2014.
} 
${ }^{6}$ Técnicos jurídicos y otros operadores de justicia administrativos.

${ }^{7}$ Dependiendo del tribunal observado en el caso de aquel situado en el sector sur de la capital, éramos nosotros los encargados de seleccionar una mujer explicar por qué nos acercábamos a ella y consultar si era posible acompañarlas durante la duración de su estancia en la mañana en el Tribunal de Familia. En el tribunal del sector nor-oriente de la capital, nosotros acompañábamos a un profesional del área psicosocial - Consejeros Técnicos- quienes normalmente tenían a personas citadas debido a una interposición de denuncia de manera previa a través de la Policía o por vía telefónica. De todos modos, las mujeres se elegían de manera aleatoria y siempre se les consultó sobre su disponibilidad a ser parte del estudio.

${ }^{8}$ Esta experiencia si bien fue importante no tuvo la misma cantidad de dedicación en tiempo por problemas de coordinación entre el Fiscal y la investigadora. Por tanto, se prefiere detallar en otras instancias a través de entrevistas la implementación de la política pública en el sector penal.

${ }^{9}$ Cada tribunal elabora en conjunto con sus profesionales una pauta de medición de riesgo para los casos de violencia intrafamiliar. Pauta que permite establecer un filtro para seleccionar los casos y su posterior audiencia dependiendo del riesgo de la víctima o de las víctimas.

${ }^{10}$ Se considera por parte de la legislación chilena un auxiliar de la justicia a aquellos profesionales y funcionarios administrativos no formados en derecho.

\section{Bibliografía}

Azócar, M. J. y Ferree, M.M. (2015). Gendered expertise, Gender \& Society, 29 (6), 841-862.

Bourdieu, P. (1986). La force du droit: Éléments pour une sociologie du champ juridique, Actes de la recherche en sciences sociales, (64), 3-19.

Blin, Th. (1999). Phénoménologie de l’Action Sociale à partir d’Alfred Schütz. Paris, France: L’Harmattan.

Commaille, J. (2010). Les vertus politiques du droit. Mythes et réalités, Droit et société, 3 (76), 695-713.

Coman, R., Crespy, A., Louault, F., Morin, J. F., Pilet, J.B y van Haute, E. (2016). Méthodes de la science politique. De la question de départ à l'analyse des données, Louvain- la- Neuve: De Boeck Supérieur.

Crenshaw, K. (1989). Demarginalizing the intersection of race and sex: A black feminist critique of antidiscrimination doctrine, feminist theory and antiracist politics. U. Chi. Legal F.

Chauvin, S.n y, Jounin, N. (2012). «L’observation directe», in Paugam, S. (Dir.). L enquête sociologique (pp. 143165) París, France: Presses Universitaires de France

Delpeuch, Th., Dumoulin, L. y de Galembert, C. (2014). Sociologie du droit et de la justice. París, France: Armand Colin.

Dodier, N. y Baszanger, I. (1997). Totalisation et altériteì dans l'enquête ethnographique, Revue française de sociologie, 38 (1), 37-66.

Dubois, V. (2018). El burocráta y el pobre. Relación administrativa y tratamiento de la miseria. Valencia, España: Institució Alfons el Magnànim.

Dupret, B. (2010). Droit et sciences sociales. Pour une respécification praxéologique, Droit et société, 2 (75): 316.

Franzé Mudanó, A. (2013). Perspectivas antropológicas y etnográficas de las políticas públicas, Revista de Antropología Social, (22), 9-23.

Glaser, B.G. y Strauss, A.L. (1967). The discovery of grounded research: strategies for qualitative research. New Brunswick (USA) and London (UK): Aldine Transaction. 
Hammersley, M. y Atkinson, P. (2007). Ethnography. Principles in practice. London et New York: Routledge.

Hersant, J. (2017). Patronage and Rationalization: Reform to Criminal Procedure and the Lower Courts in Chile, in Law \& Social Inquiry, 42 (2), 423-449.

Jobert, B. y Muller, P. (1987). L’État en action: politiques publiques et corporatismes. Paris, France: Presses universitaires de France.

Joseph, L., Mahler, M. y Auyero, J. (eds.) (2007). New perspectives in political ethnography. New York, USA: Springer.

Le Caisne, L. (2007). De si dangereux condamnés. Journal des anthropologues, (1), 183-210.

Mazouz, S. (2011). La République et ses autres: politiques de la discrimination et pratiques de naturalisation dans la France des années 2000 (Tesis de doctorado). EHESS, París.

Mazouz, S. (2014). Ni juridique, ni politique. L’anti-discrimination en pratique dans une Commission pour la promotion de l'égalité des chances et la citoyenneté, Droit et société, 1 (86), 11-32.

Miranda Pérez, F. (2017). Action Publique et Justice dans le Chili post-dictatorial. Le traitement étatique des violences envers les femmes (Tesis de doctorado en Ciencia Política). Université de Grenoble Alpes, Francia.

Miranda Pérez, F., González, P. (2018). Miradas institucionales sobre la categoría de género en materia de violencias contra las mujeres en Chile. Millcayac, Revista digital en Ciencias Sociales, 5 (9), 141-162.

Miranda Pérez, F., Riveros, M.J.. s.f. Profesionales psicosociales en la justicia familiar en Chile: ¿Nuevas discrecionalidades en los espacios judiciales? working paper.

Muller, P., Leca, J., Majone, G., Thoening, J.C. y Duran, P. (1996). Enjeux, controverses et tendances de l'analyse des politiques publiques, in Revue française de science politique, 46 (1), 96-133.

Muller, P. (2000). L’analyse cognitive des politiques publiques: vers une sociologie politique de l'action publique, Revue française de science politique, 50 (2),189-208.

(2014). Référentiel, en Boussaguet, L., Jacquot, S., Ravinet, P. Dictionnaire des politiques publiques (pp. 555-562). París, France: Presses de Sciences.

Palier, B.y Surel, Y.(2005). Les «trois i» et l'analyse de l’État en action, Revue française de science politique, 55 (1), 7-32.

Peneff, J. (2009). Le goût de l'observation. Comprendre et pratiquer l'observation participante en sciences sociales. París, France: La Découverte, coll.

Revuelta, B. (2007). La implementación de políticas públicas, Díkaion, 16 (1), 135-146.

Rojas Lasch, C. (2014). Sexuación y subjetivación en las prácticas de asistencia en Chile, Cadernos de Pesquisa, 44 (152), 312-333.

Rojas Lash, C. (2019). Ayudar a los pobres. Etnografía del Estado Social y las prácticas de asistencia. Santiago, Chile: Ediciones Alberto Hurtado.

Sabatier, P. y Mazmanian, D. (1980). The implementation of public policy: A framework of analysis, Policy studies journal, 8 (4), 538-560. 
Subirats, J., Knoepfel, P., Larrue, C. y Varone, F. (2008). Análisis y gestión de políticas públicas. Barcelona, España: Editorial Ariel.

Surel, Y. (2010). Approches cognitives, in Boussaguet, L., Jacquot, S., Ravinet, P., Dictionnaire des politiques publiques (pp.90-98). París, France: Presses de Sciences

Spire, A. (2005). Étrangers à la carte. L administration de l immigration en France (1945-1975). París, France: Grasset.

Sharma, A., y Gupta, A. (2006). Rethinking Theories of the State in an Age of Globalization, in Shrama y Gupta (eds), The Anthropology of the State. A reader (pp. 1-41) Mylon Keynes: Blacwell:

Schatz, E. (Éd.) (2013). Political ethnography: What immersion contributes to the study of power. Chicago, USA: University of Chicago Press.

Villegas Díaz, M. (2012). El delito de maltrato habitual en la Ley N²0.066 a la luz del derecho comparado, Política Criminal, 7 (14), 276-317.

Weber, M. (2006). Conceptos sociológicos fundamentales. Madrid, España: Alianza Editorial. 